Cockcroft-11-36

Low Energy Storage Rings: Opening Routes for Beyond State-of-the-art Research

Carsten P. Welsch 


\section{AIP Conference Proceedings}

\section{Low Energy Storage Rings: Opening Routes for Beyond Stateoftheart Research}

Carsten P. Welsch

Citation: AIP Conf. Proc. 1370, 40 (2011); doi: 10.1063/1.3638080

View online: $\mathrm{http}: / / \mathrm{dx}$.doi.org/10.1063/1.3638080

View Table of Contents: http://proceedings. aip.org/dbt/dbt.jsp?KEY=APCPCS\&Volume $=1370 \& \mid s s u e=1$

Published by the American Institute of Physics.

\section{Related Articles}

Non-resonant parametric amplification in biomimetic hair flow sensors: Selective gain and tunable filtering Appl. Phys. Lett. 99, 213503 (2011)

The effect of electrostatic shielding using invisibility cloak

AlP Advances 1, 042126 (2011)

A molecular Debye-Hückel theory and its applications to electrolyte solutions

J. Chem. Phys. 135, 104104 (2011)

Adhesion selectivity by electrostatic complementarity. I. One-dimensional stripes of charge

J. Appl. Phys. 110, 054902 (2011)

Quantitative potential measurements of nanoparticles with different surface charges in liquid by open-loop electric potential microscopy

J. Appl. Phys. 110, 044315 (2011)

\section{Additional information on AIP Conf. Proc.}

Journal Homepage: http://proceedings.aip.org/

Journal Information: http://proceedings.aip.org/about/about_the_proceedings

Top downloads: http://proceedings.aip.org/dbt/most_downloaded.jsp?KEY=APCPCS

Information for Authors: http://proceedings.aip.org/authors/information_for_authors

\section{ADVERTISEMENT}

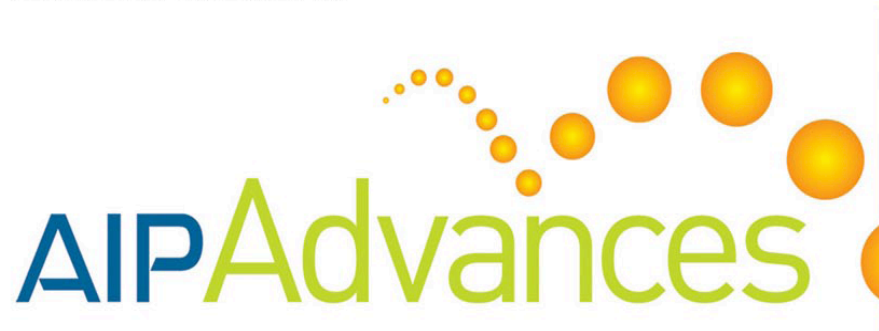

Submit Now

\section{Explore AIP's new \\ open-access journal}

Article-level metrics now available

Join the conversation!

Rate \& comment on articles 


\title{
Low Energy Storage Rings: Opening Routes for Beyond State-of-the-art Research
}

\author{
Carsten P. Welsch ${ }^{\mathrm{a}, \mathrm{b}}$ \\ ${ }^{a}$ Cockcroft Institute, Daresbury Science and Innovation Campus, WA4 4AD Warrington, UK \\ ${ }^{b}$ University of Liverpool, Physics Department, L69 7ZE Liverpool, UK
}

\begin{abstract}
Electrostatic storage rings have proven to be invaluable tools for atomic and molecular physics at the ultra-low energy range from 1 to $100 \mathrm{keV} / \mathrm{A}$. Due to the mass independence of the electrostatic rigidity these machines are able to store a wide range of different particles, from light ions to heavy singly charged bio-molecules. Their beam dynamics is, however, fundamentally different to magnetic storage rings and therefore needs to be investigated in detail to optimize storage ring performance and experimental output. This paper first gives an overview of existing electrostatic storage rings and their experimental programs. Second, future machines in Heidelberg, Stockholm and the Facility for Antiproton and Ion Research (FAIR) are described and the main challenges are summarized. Finally, the focus is set on a flexible storage ring facility presently being built up at the King Abdulaziz Center for Science and Technology (KACST) in Riyadh, Saudi Arabia, that addresses a broad user community and will allow for a next-generation experimental program in the low energy regime.
\end{abstract}

Keywords: Storage rings, electrostatic fields, atomic physics, antimatter research

PACS: 29.20.Ba , 29.20.D-, 41.75.-i, 41.85.-p

\section{INTRODUCTION}

There are more than fifteen thousand particle accelerators in the world, from the linear accelerators used for cancer therapy in modern hospitals to the giant 'atom-smashers' at international particle physics laboratories used to unlock the secrets of creation. For many decades these scientific instruments have formed one of the main pillars of modern research across scientific disciplines and countries.

Magnetic storage rings have been an invaluable tool for atomic, nuclear and high energy physics since the late 1960s. While the main goal has been for a long time to reach higher beam energies, developments since the $1980 \mathrm{~s}$ have concentrated on specific research areas in the middle and low energy range. Storage rings, such as for example the ESR at GSI (Germany), ASTRID at ISA (Denmark), and CRYRING at MSL (Sweden), have allowed for storing exotic ions over extended periods of time, for studying their controlled interaction with laser and particle beams, and for the development of new beam handling and cooling techniques [1-5].

A new type of storage ring has given access to previously inaccessible very low beam energies in the tens of keV range since the year 1998, when ELISA started operation [6]. This storage ring relies on electrostatic elements for both, beam bending and modulation. The main difference to magnetic storage rings is that the electrostatic rigidity $E \cdot \rho$ is mass-independent. This enables dynamic experiments with heavy bio molecules with atomic masses of up to even thousands of amu at very low beam energies that cannot be carried out in any magnetic ring. Most of the building blocks of life, such as for example proteins, amino acids or even DNA have never been analyzed dynamically. In such a ring, merged electron or laser beams can be used to prepare these ions into specific states of interest or to bring them into systematic fragmentation. Combined with the state-of-the art imaging techniques [7], such storage rings can then give access to measurements of the dynamic correlation in complex systems with unprecedented resolution.

In contrast to single pass experiments, where the ion beam passes only once through the target, the ions can be stored for millions of turns in such a setup. This improves the achievable event rates by many orders of magnitude and thus allows to record the momenta of all neutral particles and to thereby draw a complete picture of the reaction of interest.

Proceedings of the Fifth Saudi Physical Society Conference

AIP Conf. Proc. 1370, 40-46 (2011); doi: 10.1063/1.3638080

(C) 2011 American Institute of Physics 978-0-7354-0930-9/\$30.00 


\section{Principles of Electrostatic Storage Rings}

An ion storage ring consists of bending elements to keep the ions on a cyclic orbit and focusing elements to modulate the ion beam in the machine. At high beam energies, close to the speed of light, the relation between magnetic and electric field is $E[\mathrm{~V} / \mathrm{m}]=c B[T]$. Therefore, to generate as similar force on a charged particle as caused by a magnetic field strength of $1 \mathrm{~T}$, one needs an electric field of $310^{8} \mathrm{~V} / \mathrm{m}$. While such magnetic field strength can be realized with conventional magnet technology, typical electric fields are limited to much lower values because of field breakdown.

At lower beam energies, however, it becomes increasingly difficult to generate magnetic fields that are homogeneous enough to keep the ions on a stable orbit over many revolutions in a storage ring. For a machine that shall be operated at different beam energies around some tens of $\mathrm{keV}$, remanence and hysteresis effects in magnets are problems that are very difficult to overcome. In such case, electrostatic fields with the necessary homogeneity are much easier to generate, even at lowest beam energies.

As mentioned in the introduction, the main advantage from using electrostatic ion lenses, is the fact that the electrostatic rigidity is mass-independent. Therefore, two beams of particles at similar charge state and total energy can be stored with an identical field configuration. This means for example that an electrostatic storage ring can be setup with an intense proton beam, before turning to the possibly much more difficult to generate beam of interest.

\section{Beam Bending}

In the simplest configuration, two parallel electrodes can be used for deflecting a charged particle beam. Inside such deflector, the beam will follow a parabolic path. The actual deflection will depend on the beam's kinetic energy, charge state and the strength of the electric field, i.e. the voltages on the electrodes and the distance between them.

Such deflectors have been used in the existing storage rings in Denmark, Japan and in Germany. As the voltages on the plates can be switched on and off rather quickly, such deflectors also offer a convenient way for injecting the ion beam into the ring. Their use, however, is limited to rather small deflecting angles, as the necessary distance between the plates to allow deflection - and hence the voltages on the plates - grow quickly. Moreover, even the design particle gets accelerated or decelerated inside this deflector type, as the zero Volt potential line lies in the middle between both plates and hence does not coincide with the ion path.

Larger bending angle are realized by bend electrodes of either cylinder or spherical shape. The latter leads to a focusing effect in both transverse planes and hence generates a narrow waist. It was shown in recent studies that the non-linear fields inside a spherical deflector strongly reduce the available dynamic aperture and hence this geometry is not the optimum choice for a storage ring [8]. Both deflector geometries allow for letting the zero Volt potential coincide with the design orbit. This eliminates the unwanted effect described in the previous paragraph and is the reason why energy variable rings, such as the CSR and USR described in later sections, use cylinder deflectors also for small angle deflection in the ring corners.

It should be pointed out that there is still beam acceleration and deceleration in each bending element. This is caused by the entrance and exit fringe fields and cannot be eliminated. This coupling between the longitudinal and transverse phase space is a particular difference in the beam dynamics as compared to magnetic storage rings.

\section{Transverse Beam Modulation}

The transverse size of the beam is modulated by electrostatic quadrupole doublets. Ideally, electrodes of hyperbolic shape are used as they generate a force on the beam that goes linear with the distance of a particle from the design orbit. All electrodes are normally on the same potential with opposite electrodes being either both, positively or negatively charged, as shown in figure 1 .

As the manufacture of hyperbolic electrode surfaces is a lengthy and complicated procedure, cylinder electrodes with radius $r=1.14 r_{a p}$ are also used, as they best approximate the ideal field distribution. This is particularly true for low energy beam transport lines in which case the slight deviations caused by the cylinder electrodes is not of relevance as the ions pass only once through them. 


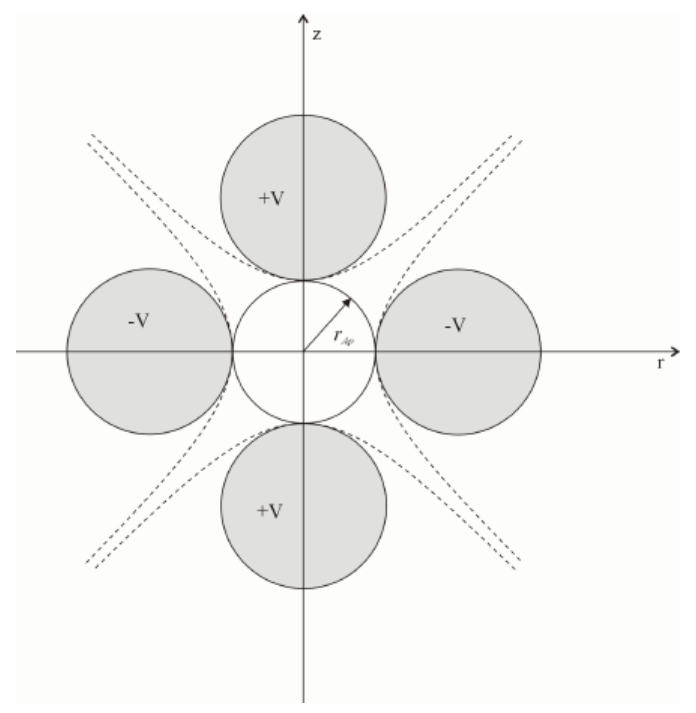

FIGURE 1. Cross section of an electrostatic quadrupole.

\section{OVERVIEW OF ELECTROSTATIC STORAGE RINGS}

An overview of existing and planned electrostatic storage rings is given in table 1. Besides a proof-of-principle machine built in 1953 that was used to demonstrated the principle of alternate gradient focusing for the Brookhaven synchrotron, the electrostatic ring ELISA was the first ever electrostatic ring. It started operation in 1998 and has stored many different ion species during the last decade $[9,10]$. Two storage rings with a similar lattice were built in Japan at Tokyo Metropolitan University and at KEK [11,12]. All three rings operate at a fixed energy of up to 25 $\mathrm{keV} \cdot \mathrm{q}$. Various decay processes and life times of instable ions have been analyzed in these facilities and different crossed and merged beam experiments have been realized.

TABLE 1. Overview of electrostatic storage rings around the world.

\begin{tabular}{|c|c|c|c|c|c|c|c|c|}
\hline $\begin{array}{c}\text { Ring } \\
\text { Achronym }\end{array}$ & ELISA & $\begin{array}{c}\text { TMU + } \\
\text { KEK }\end{array}$ & FLSR & DESIREE & CSR & USR & AD Rec & $\begin{array}{l}\text { Pro- } \\
\text { ESR }\end{array}$ \\
\hline Location & $\begin{array}{c}\text { Aarhus, } \\
\text { Denmark }\end{array}$ & $\begin{array}{c}\text { Tokyo and } \\
\text { Tsukuba, } \\
\text { Japan }\end{array}$ & $\begin{array}{l}\text { Frankfurt, } \\
\text { Germany }\end{array}$ & $\begin{array}{l}\text { Stockholm, } \\
\text { Sweden }\end{array}$ & $\begin{array}{c}\text { Heidelberg, } \\
\text { Germany }\end{array}$ & $\begin{array}{c}\text { Darmstadt, } \\
\text { Germany }\end{array}$ & $\begin{array}{c}\text { Geneva, } \\
\text { Switzerlan } \\
\text { d }\end{array}$ & $\begin{array}{c}\text { Riyadh, } \\
\text { Saudi } \\
\text { Arabia }\end{array}$ \\
\hline Ion Species & $\mathrm{A}<100$ & $\mathrm{~A}<100$ & $\mathrm{~A}<100$ & $\mathrm{~A}<100$ & $\mathrm{~A}<100$ & Pbars & Pbars & $\mathrm{A}<100$ \\
\hline $\begin{array}{l}\text { Energy } \\
{[\mathrm{keV}]}\end{array}$ & $(5-25) \mathrm{q}$ & $20 q$ & $50 \mathrm{q}$ & $(25-100) \dot{q}$ & $(300-20) \cdot \mathrm{q}$ & $(300-20) \mathrm{q}$ & $(3-30) \cdot q$ & $30 q$ \\
\hline Symmetry & 2 & 2 & $2 / 4$ & 2 & 4 & 4 & 2 & 2 \\
\hline Circum. [m] & 6.3 & 8.1 & 14.2 & $2 \times 9.2$ & 35 & 43 & 8 & 9.2 \\
\hline Deflectors & $160^{\circ}+10^{\circ}$ & $160^{\circ}+10^{\circ}$ & $75^{\circ}+15$ & $160^{\circ}+10^{\circ}$ & $39^{\circ}+6^{\circ}$ & $37^{\circ}+8^{\circ}$ & $90^{\circ}+90^{\circ}$ & $166^{\circ}+7^{\circ}$ \\
\hline e-cool & No & No & No & No & Yes & Yes & No & No \\
\hline $\begin{array}{l}\text { Vacuum } \\
\text { [mbar] }\end{array}$ & $10^{-11}$ & $10^{-11}$ & $10^{-11}$ & $10^{-13}[10 \mathrm{~K}]$ & $10^{-15}[10 \mathrm{~K}]$ & $10^{-11}$ & $10^{-10}$ & $10^{-10}$ \\
\hline Modes & Storage & Storage & low $\beta, D=0$ & $\begin{array}{c}\text { Merged/ } \\
\text { colliding } \\
\text { beams }\end{array}$ & $\begin{array}{l}\text { Cooled } \\
\text { beam, } \\
\text { storage }\end{array}$ & $\begin{array}{c}\text { Short } \\
\text { bunches, } \\
\text { extraction }\end{array}$ & $\begin{array}{l}\text { low } \beta, \\
\text { low D }\end{array}$ & Storage \\
\hline Status & Running & Running & Tests & Assembly & Assembly & Design & $\begin{array}{l}\text { Manu- } \\
\text { facture }\end{array}$ & $\begin{array}{l}\text { Manu- } \\
\text { facture }\end{array}$ \\
\hline
\end{tabular}

A larger storage ring was designed and built up at Frankfurt University. It allows for storing ions up to an energy of $50 \mathrm{keV} \cdot \mathrm{q}$ [13-15]. Although initially built up in a racetrack layout, the ring was designed with a fourfold symmetry in mind to allow for the installation of a larger number of internal experiments. 
A significant step forward is the cooling of the whole ring to temperatures below $10 \mathrm{~K}$. This substantially reduces the residual pressure in the machine and hence helps increasing the beam life time. It also gives access to experiments with ro-vibrationally cooled ions.

The double ring DESIREE, which is presently being assembled at Stockholm University in Sweden, has a unique layout in that it combines two almost identical storage ring in a very large vacuum vessel [16]. This will allow for merged and colliding beams experiments.

The cryogenic storage ring (CSR), which is presently being assembled at the Max Planck Institute for Nuclear Physics in Heidelberg, will be the first-ever energy variable cooler synchrotron [17,18]. It will allow varying the beam energy from $300 \mathrm{keV}$ down to $20 \mathrm{keV}$ and will operate at both, room temperature and below $10 \mathrm{~K}$. At the lowest beam energy, the cooler needs to provide a beam of cold electrons at an energy of $10 \mathrm{eV}$ only, which is a new operating regime for electron coolers and a significant challenge for this project. Commissioning of the ring is expected to start in 2012 .

\section{The Ultra-low Energy Storage Ring (USR)}

Antiprotons, stored and cooled at low energies, in a storage ring or at rest in traps are highly desirable for the investigation of a large number of basic questions on fundamental interactions, on the static structure of exotic antiprotonic atomic systems and (radioactive) nuclei and on the time-dependent quantum dynamics of correlated systems. However, such cooled beams with the necessary beam characteristics for these fundamental studies are presently not available. Therefore, a novel electrostatic cooler synchrotron, the Ultra-low energy Storage Ring (USR) $[19,20]$ and a state-of-the-art in-ring spectrometer, based on the reaction microscope principle, are being developed in the QUASAR group [21]. This machine shall form a central part of the future Facility for Low energy Antiproton and Ion Resarch (FLAIR) in Germany. The USR shall provide cooled beams of antiprotons at energies between 20 and $300 \mathrm{keV}$.

In order to match the requirements from the envisaged experiments, different modes of operation have to be included in the USR: deceleration and electron cooling, fast and slow extraction, in-ring experiments with ultra-short antiproton pulses, and optimization of the beam shape, size, and dispersion with respect to the respective internal or external experiment.

The USR lattice is based on a split-achromat geometry, which gives the necessary flexibility to satisfy the before-mentioned boundary conditions. The storage ring has a circumference of $42.6 \mathrm{~m}$ and consists of four $4 \mathrm{~m}$ long straight sections that are used to accommodate a reaction microscope, the different $\mathrm{rf}$ systems for a particular short bunch operation mode [22,23], an electron cooler, a decelerating drift tube, and elements for fast/slow extraction $[24,25]$. In addition, one of the straight sections is kept free for the possible inclusion of a merged positron ring, see [26]. Each quarter contains five electrostatic quadrupoles, two $8^{\circ}$ and two $37^{\circ}$ electrostatic deflectors and a drift space of $1 \mathrm{~m}$ between the $8^{\circ}$ and the $37^{\circ}$ deflectors to allow for the detection of neutral particles leaving the ring with an opening angle of up to $\Omega=+/-0.7^{\circ}$ after the reaction microscope.

Very short beam pulses in the nanosecond regime are formed in a combination of $\mathrm{rf}$ techniques: First, the beam is captured into $50 \mathrm{~ns}$ rf buckets by a $20 \mathrm{MHz}$ cavity. The ultra-short pulses of 1-2 ns duration are then formed by an additional $3 \beta \lambda / 2$ double drift buncher with a voltage of a few hundreds of Volts. The buncher for phase compression is located at the beginning of one of the straight sections of the USR. The very short bunches are then formed in the symmetry point of the straight section where the reaction microscope is located and where the dispersion function and its first derivative are both zero. Once this experimental section is crossed, an rf phase de-compressor reduces the energy spread introduced by the buncher to an acceptable level.

A slow and fast extraction has been co-located in the same straight section of the USR. The beam extraction system consists of two parallel plate deflectors with a gap of $60 \mathrm{~mm}$, two slightly larger parallel plate deflectors with a gap width of $90 \mathrm{~mm}$, a $6^{\circ}$ extraction septum and a $30^{\circ}$ cylinder deflector. The electrostatic septum which is located in-between the two central bump electrodes is displaced by $50 \mathrm{~mm}$ from the central orbit to provide sufficient free space to accommodate both, the circulating and the extracted beam.

During the extraction process, the beam is first moved slowly by a local orbit bump towards the extraction septum. This is realized by a local orbit bump created by four dedicated parallel plate electrostatic deflectors. The first electrode bends the beam by an angle between $4.5^{\circ}$ and $6^{\circ}$. The second larger bump electrode returns the beam to its initial direction. The beam is then parallel to the equilibrium orbit but shifted away from the ring axis by $30-40$ $\mathrm{mm}$ during the passage of the $1240 \mathrm{~mm}$ long part of the straight section. A second pair of bump electrodes directs the beam back on the ring axis. This scheme moves the stored ion beam close to the septum, thus reducing the necessary voltages on the extraction elements and giving more space for the circulating beam. In fast extraction, an 
additional pulsed voltage of opposite polarity is applied to the plates of the large bump deflector. This switches the electrode off and allows the beam to pass directly into the electrostatic septum deflector.

Slow extraction will be realized by using a third-integer resonance [25]. This will allow for a quasi-continuous beam that can be delivered to the different experimental areas. As soon as the resonance-driving sextupoles are powered, the stable phase-space ellipse shrinks and gets distorted, creating a separatrix area of triangular shape where the radial motion is distorted but still stable. For larger amplitudes of the radial oscillations, particles now leave the stable area and follow the separatrix arms. This will move the particles towards the vacuum chamber and thus into the extraction electrode.

A small antiproton recycling ring has been designed recently as a prototype of the USR. This very compact storage ring uses 50\% scaled versions of the USR ion optical elements and will allow for measuring for the first time differential cross section in collisions between antiprotons and different target gases [27].

\section{An Electrostatic Storage Ring at KACST}

A state-of-the-art fixed energy electrostatic storage ring that will allow for precision experiments with most different kinds of ions in the energy range of up to $30 \mathrm{keV}$ will be constructed and operated at the National Centre for Mathematic and Physics (NCMP) at the King Abdulaziz City for Science and Technology (KACST). The ring shall serve as the central machine of a unique and highly flexible experimental platform. The lattice design therefore has to cover the different experimental techniques that the ring will be equipped with, such as e.g. electron-ion crossed-beams and ion-laser/ion-ion/ion-neutral merged-beams techniques.

This racetrack-type storage ring has a circumference of $9.2 \mathrm{~m}$ and consists of two large $166^{\circ}$ cylinder deflectors, accompanied by two $7^{\circ}$ parallel plate deflectors, which will also be used during the injection process. Electrostatic quadrupoles are used for beam modulation. The respective dimensions of all ion optical elements are comparable to the ones at ELISA [28].

A highly flexible injector complex will be used to inject ions from different sources into the ring [29]. It is planned to add a magnet selector for high mass resolution in the injector at a later stage and even the addition of a second ring for merged beam experiments has been included in the ring design.



FIGURE 2. Overview of the low energy ion generation and storage complex at KACST. Beams from different ion sources can be injected into the electrostatic ring shown on the left.

The beam instrumentation for low intensity beams with below $10^{7}$ particles at energies of only some tens of keV poses many challenges and requires an $\mathrm{R} \& \mathrm{D}$ program into beam diagnostics. The beam diagnostics system of the 
ring at KACST can serve as an illustration of the developments that have been realized for the latest electrostatic ring projects.

Non-destructive beam position measurement is realized by means of capacitive pick-ups. Diagonal-cut electrodes are used to generate an output signal that is linear with beam displacement inside the pick-up. Grounded electrodes at the entrance and exit of the detector and in-between electrode segments limit signal cross-talk and optimize signal-to-noise levels. The design of such pick-ups was also optimized with respect to the surrounding vacuum chambers to avoid beam instabilities due to beam-to-ground impedance jumps [30,31].

The measurements of the transverse beam profile is normally realized either via the detection of neutral particles at the end of the straight sections or by a scintillator-based monitor. Limited sensitivity and light yield decrease due to surface sputtering have been reported [32,33] for such monitors that triggered additional tests. Experiments at the Nuclear Physics Laboratory INFN-LNS in Catania, Italy were realized recently that were based on irradiation of different screens with a continuous beam of protons in the $\mathrm{keV}$ range with intensities down to a few fA. The scintillating materials used during the investigations included CsI:Tl, YAG:Ce and a Tb-glass-based Scintillating Fibre Optic Plate (SFOP). In order to reduce the initial beam currents of a few pA to only a few fA, pepper-pot-like attenuators were used, which produced multi-peak images. This allowed resolution testing of the screens at the same time. Results indicated a high sensitivity to low intensity, low energy beams such as those expected from most present and future electrostatic rings. For $200 \mathrm{keV}$ protons, the beam was still visible at approx. $10 \mathrm{fA}$ and only a few seconds of averaging with a resolution better than $0.5 \mathrm{~mm}$ [34].

In addition to such a scintillator-based monitor, an ionization beam profile monitor relying on a supersonic gasjet shaped into an extended thin curtain can be used for monitoring the transverse beam profile $[35,36]$. Existing inring monitoring techniques, such as residual gas monitors, can take up to about $100 \mathrm{~ms}$ [37] to make meaningful measurements due to the low residual gas pressure. The curtain jet monitor allows for varying the gas density and thus the event rate and for a two dimensional transverse profile measurement as well as beam imaging [38,39].

This monitor relies on a neutral gas-jet, shaped into a thin curtain crossing the beam. In its simplest configuration, the gas curtain crosses the stored ion beam under an angle of $45^{\circ}$. While the stored beam crosses the jet, ionization occurs. These ions are then accelerated by an electric extraction field towards an amplification stage with an MCP and detected via a phosphor screen and a CCD camera.

\section{SUMMARY AND OUTLOOK}

Existing electrostatic storage rings have been used for many interesting and novel experiments, profiting directly from the accessible very low energies and the mass independence of the electrostatic rigidity. Future projects build up on this successful base, but aim at providing electron cooled beams at variable beam energies, with highly flexible beam characteristics. This will allow further establishing electrostatic storage rings as true multi-purpose, multi-user facilities.

\section{ACKNOWLEDGMENTS}

The generous support of the Helmholtz Association of National Research Centers under contract no. VH-NG-328 and the GSI Helmholtz Centre for Heavy Ion Research is acknowledged. The kind invitation by the Saudi Physical Society to present this work at the SPS5 conference is acknowledged.

\section{REFERENCES}

[1] B. Franzke, "Information about ESR parameters", GSI-ESR-TN/87-02.

[2] P. Lefèvre, D.Möhl, “A Low Energy Accumulation Ring of Ions for LHC”. CERN/PS 93-62(DI) 259 (1993).

[3] S. Moeller, "The Aarhus Storage Ring for Ions and Electrons ASTRID”. Proc. Part. Acc. Conf., Washington, USA (1993).

[4] E. Jaeschke et al., "The Heidelberg Test Storage Ring for Heavy Ions TSR", Proc. Europ. Part. Acc. Conf., Rome, Italy (1988).

[5] M. Grieser et al., "Recent Developments at the TSR Heidelberg”, Proc. Europ. Part. Acc. Conf, London, UK (1994).

[6] S.P. Moeller, ELISA - an Electrostatic Storage Ring for Atomic Physics, Proc. European Part. Acc. Conf., Stockholm, Sweden (1998).

[7] J. Ullrich et al, "Recoil-Ion and electron momentum spectroscopy: reaction-microscopes", Rep. Prog. Phys. 66 (2003) 1463.

[8] A.I. Papash, O. Gorda, C.P. Welsch, "Simulations of Space Charge effects in Low Energy Electrostatic Storage Rings". Proc. Int. Part. Accel. Conf., Kyoto, Japan (2010). 
[9] S.P. Moeller, "Design and First Operation of the Electrostatic Storage Ring ELISA", Proc. Europ. Part. Acc. Conf., Stockholm, Sweden (1998).

[10] S.P. Moeller et al., "Operational Experience with the Electrostatic Storage Ring, ELISA", Proc. Part. Acc. Conf., New York, USA (1999).

[11] T. Tanabe et al., An Electrostatic Storage Ring for Atomic and Molecular Science, Nucl. Instr. And Meth. A 482 (2002) $595 \mathrm{c}$.

[12] S. Jinno et al., TMU electrostatic ion storage ring designed for operation at liquid nitrogen temperature, Nucl. Instr. and Meth. A $532(2004) 477 \mathrm{c}$.

[13] C.P. Welsch et al.,An Electrostatic Storage Ring as the Central Machine of the Frankfurt Ion Storage Experiments, PRSTAB 7, 080101 (2004).

[14] C.P. Welsch et al., "FIRE—-the Frankfurt Ion stoRage Experiments", Nucl. Instr. and Meth. A 527 (2004) 284cc.

[15] K.E. Stiebing et al., "FLSR - The Frankfurt Low Energy Storage Ring”, Nucl. Instr. Meth. A 614 (2010).

[16] K.-G. Rensfelt et al.,DESIREE - a Double Electrostatic Storage Ring, Proc. Europ. Part. Acc. Conf., Lucerne, Switzerland (2004).

[17] D.Zaifman, et al., "Physics with colder molecular ions: The Heidelberg Cryogenic Storage Ring CSR". Journal of Physics. Sixth Int. Conf. on Dissoc. Recombination. V.4, Iss.1. (2005).

[18] R. von Hahn et al., "CSR - a Cryogenic Storage Ring at MPI-K", Proc. Europ. Part. Acc. Conf., Lucerne, Switzerland (2004).

[19] C.P. Welsch, et al., "An ultra-low-energy storage ring at FLAIR", NIM A 546 (2005) 405-417.

[20] A.I. Papash, C.P. Welsch, "An Update of the USR Lattice: Towards a true multi-user experimental facility", Proc. Part. Acc. Conf., Vancouver, Canada (2009).

[21] http://www.quasar-group.org.

[22] A.I. Papash, C.P. Welsch, "On the Possibility of Realizing Shortest Bunches in Low-Energy Storage Rings", Physics of Particles and Nuclei Letters, Vol. 6, No. 3., p.216-226 (2009).

[23] A.I. Papash, C.P. Welsch, "Ultra-short Pulse Operation Mode of the Ultra-low Energy Storage Ring", Nucl. Instr. Meth. A 620, p.128-141 (2010)

[24] P. Schmid, et al., "Preliminary Design of an Extraction Scheme for the USR", Hyperfine Interact. 194 1-3 p. 183-187 (2009).

[25] G.A. Karamisheva, AI.Papash, C.P.Welsch, "Study of Slow and Fast Extraction for the Ultralow Energy Storage Ring", Physics of Particles and Nuclei Letters, accepted (2010).

[26] E.Ahmanova et al., "Advances in the LEPTA Project”, Proc. Russian Part. Acc. Conf., Protvino, Russia (2010).

[27] M.R.F. Siggel-King et al., "Electrostatic Low-Energy Anti- proton Recycling Ring". Hyperfine Interactions, submitted (2010).

[28] M.A. El Ghazaly et al., "Layout of a Novel Electrostatic Storage Ring at KACST", Applied Mathematics \& Information Sciences 3 (3) (2009), 309-319.

[29] M.A. El Ghazaly et al., "A Highly Flexible Low Energy Ion Injector at KACST“, Proc. Part. Acc. Conf., Vancouver, Canada (2009).

[30] J. Harasimowicz, C.P. Welsch, "Beam Instrumentation for the Future Ultra-low Energy Storage Ring at FLAIR", Hyperfine Interact. 194 1-3 p. 177-181 (2009).

[31] J. Harasimowicz, C. P. Welsch, "Optimisation Studies of a Resonant Capacitive Pick-Up for Beam Position Monitoring of Low Intensity, Low Velocity Antiproton Beams at FLAIR”, Proc. DIPAC'09, Basel, Switzerland, p. 300-302 (2009).

[32] T. Sieber et al., "A Beam Diagnostics System for the Heidelberg Cryogenic Storage Ring CSR", Proc. Europ. Part. Acc. Conf., Edinburgh, Scotland (2006).

[33] M. Vogel et al., "Scintillation Light Produced by Low-energy Beams of Highly-charged Ions", Nucl. Instrum. Meth. B 263(2), 518-522 (2007).

[34] J. Harasimowicz, et al., "Scintillating Screens Sensitivity and Resolution Studies for Low Energy, Low Intensity Beam Diagnostics", Rev. Sci.Instr. 81 (9) (2010).

[35] K.-U. Kühnel, et al., "Putignano, C.D. Schröter, J. Ullrich, C.P. Welsch, "A Novel Beam Profile Monitor Based on a Supersonic Gas Jet", Proc. Europ. Part. Acc. Conf., Genoa, Italy (2008).

[36] M. Putignano, et al., "A fast, low perturbation ionization beam profile monitor based on a gas-jet curtain for the ultra low energy storage ring ", Hyperfine Interact. 194 1-3 p. 189-193 (2009).

[37] T. Honma et al., "Design and Performance of a Nondestructive Beam-profile Monitor Utilizing Charge-division Method at HIMAC", Nucl. Instr. Meth. A 490(3), 435-443 (2002).

[38] Y. Hashimoto et al., "Oxygen Gas-sheet Beam Profile Monitor for the Synchrotron and Storage Ring", Nucl. Instr. Meth. A 527(3), 289-300 (2004).

[39] M. Putignano, et al., "Design of a Nozzle-Skimmer System for a Low Perturbation Ionization Beam Profile Monitor", Proc. DIPAC Workshop, Basel, Switzerland (2009). 